\title{
Implementation of hyperfine coupling in all-electron mixed basis approach
}

\author{
Hiroyuki Terada, ${ }^{1}$ Shota Kanno, ${ }^{1}$ and Kaoru Ohno ${ }^{1, *}$ \\ ${ }^{1}$ Department of Physics, Yokohama National University, \\ 79-5 Tokiwadai, Hodogaya-ku, Yokohama 240-8501, Japan
}

(Dated: May 15, 2019)

\begin{abstract}
In order to accurately evaluate hyperfine interaction (HFI) (both the isotropic, Fermi contact term and the anisotropic, dipole interaction term) of spin-polarized atoms and molecules, we newly implement the cubic harmonics routines for calculating HFI in the all-electron mixed basis code, TOMBO, in which both plane waves (PWs) and atomic orbitals (AOs) are used as basis functions, and AOs are restricted inside the nonoverlapping atomic spheres and composed of the numerical radial function on a logarithmic radial mesh and analytic cubic harmonics. The method has a distinct merit of describing the cusp behavior near the nucleus position, which is essential for the Fermi contact term, and of significantly reducing the required cutoff energy for PWs compared to the usual PW expansion approach accompanied with the pseudopotential or APW method. We also considered the contributions from surrounding atoms in the dipole interaction. Calculated results show excellent agreement with the experimental data, indicating the excellent performance of the code.
\end{abstract}




\section{INTRODUCTION}

Hyperfine interaction (HFI) can be measured in atomic and molecular spectra using microwave ${ }^{1,2}$, electron spin resonance $(E S R)$ or electron paramagnetic resonance $(\mathrm{EPR})^{3,4}$, nuclear magnetic resonance ${ }^{5}$, Mössbauer ${ }^{6}, \gamma-\gamma$ perturbed angular correlations ${ }^{7-9}$, and so on. HFI provides explicit information on the symmetry and position of atoms, when compared with theoretical calculations for possible structures. HFI is also very important in quantum information transfer from electron spins to nuclear spins and nuclear spin entanglement ${ }^{10,11}$ as well as fine splitting of electronic energy levels in quantum qubits ${ }^{12,13}$. Although first-principles calculations of HFI have been implemented in different programs ${ }^{14-22}$, it is inevitable to accurately describe the all-electron spin density in the vicinity of nuclei. In the spin-polarized all-electron description of core orbitals, it has been emphasized that the choice of basis functions strongly influences the accuracy of the calculation; in particular, an accurate description of the cusp behavior of the Kohn-Sham orbitals at the nucleus position is essential for the isotropic term ${ }^{23}$, which is also called the Fermi contact term, in HFI. On the other hand, in the anisotropic term, which is also called the dipole interaction term, the contributions from surrounding atoms are important as well as the on-site contribution from the atom having the nuclear spin. However, such contributions have been ignored in some $\operatorname{programs}^{20-22}$.

In the all-electron mixed basis approach ${ }^{24,25}$, which has a distinct merit in describing the localized behavior as well as the extended behavior of molecular orbitals in terms of a linear combination of both plane waves (PWs) and atomic orbitals (AOs), Bahramy et al. ${ }^{22}$ first implemented the calculation routine of HFI but up to the on-site contribution only. In this approach, AOs are restricted inside the non-overlapping atomic spheres and composed of the numerical radial function on a logarithmic radial mesh and the analytic cubic harmonics. This enables us to describe the cusp behavior of the orbitals near the nuclear position. Another important merit of using this approach is that the required cutoff energy for PWs is significantly reduced from that of the usual PW expansion approach accompanied with the pseudopotential or APW method. Bahramy et al. ${ }^{22}$ used the spherical harmonics instead of the cubic harmonics for the angular part of AOs. However, for various purposes of first-principles calculations including the anisotropic contributions to HFI from surrounding atoms (present implementation), response functions (electronic and magnetic susceptibilities) using Fourier transformations $\left(\right.$ Ref. $\left.^{24}\right)$, interatomic forces $\left(\right.$ Ref. $\left.^{25}\right)$, photoelectron (or quasiparticle) and photoabsorption spectra using the $\mathrm{GW}(\Gamma)$ (+ Bethe-Salpeter 
equation) method (Refs. ${ }^{26,27}$ ), and so on, the cubic harmonics is more tractable than the spherical harmonics.

The purpose of this paper is to newly implement HFI beyond the on-site contribution using the cubic harmonics in our all-electron mixed basis code, $\mathrm{TOMBO}^{25}$, which is now applicable to various beyond DFT calculations ${ }^{26,27}$. For this purpose, we calculate explicitly the coefficients when a biproduct of the cubic harmonics is expanded in a linear combination of the cubic harmonics itself. These coefficients are used also for calculating the Fock exchange contributions from the on-site four AOs in the Hartree-Fock and GW calculations. The merit of using cubic harmonics is that the Cartesian components of the dipole interaction term in HFI can be more directly determined as well as other physical quantities in various first principles calculations. In order to crosscheck the program, we decided not to transplant Bahramy et al.'s subroutines using spherical harmonics but to make completely new subroutines using cubic harmonics. In the present paper, we will give an explicit formulation for the calculation of HFI and show the results of HFI for some atoms and small molecules, and demonstrate the ability of our method for the description of HFI.

\section{FORMULATION}

\section{A. Basic Formulation}

Hyperfine interaction between electron spin $\boldsymbol{S}$ and nuclear spin $\boldsymbol{I}_{k}$ of atom $k(k=1,2, \ldots, N)$ is described by the Hamiltonian

$H=\sum_{k}^{N} \sum_{\alpha \beta} S_{\alpha} A_{k, \alpha \beta} I_{k, \beta}$,

where $\alpha$ and $\beta$ denote $x, y, z$ components. The HFI tensor $A_{k, \alpha \beta}$ is composed of the isotropic, Fermi contact term $a_{k}$ and the anisotropic, dipole interaction term $b_{k, \alpha \beta}$ as

$A_{k, \alpha \beta}=a_{k} \delta_{\alpha \beta}+b_{k, \alpha \beta}$.

Those terms are related to the electron spin density defined as

$\rho_{s}(\boldsymbol{r})=\rho(\boldsymbol{r}, \uparrow)-\rho(\boldsymbol{r}, \downarrow)$.

The Fermi contact term is given by

$a_{k}=\frac{2}{3} \mu_{0} g_{\mathrm{e}} \mu_{\mathrm{e}} g_{k} \mu_{k} \rho_{s}\left(\boldsymbol{R}_{k}\right)$, 
where $\mu_{0}$ is the vacuum permeability, $g_{\mathrm{e}}$ is the electron $g$ factor, and $\mu_{\mathrm{e}}$ is the Bohr magneton; $g_{k}$, $\mu_{k}$, and $\boldsymbol{R}_{k}$ denote, respectively, the gyromagnetic ratio, magnetic moment, and the position of the nucleus of atom $k$. The dipole interaction term is given by

$b_{k, \alpha \beta}=\frac{1}{4 \pi} \mu_{0} g_{\mathrm{e}} \mu_{\mathrm{e}} g_{k} \mu_{k} \int \frac{\rho_{s}\left(\boldsymbol{r}_{k}+\boldsymbol{R}_{k}\right)}{r_{k}^{3}} T_{\alpha \beta}\left(\widehat{\boldsymbol{r}_{k}}\right) d \boldsymbol{r}_{k}$,

where $\boldsymbol{r}_{k}=\boldsymbol{r}-\boldsymbol{R}_{k}$ denotes the electron coordinate centered at $\boldsymbol{R}_{k}$, and $\widehat{\boldsymbol{r}_{k}}$ is the unit vector $\widehat{\boldsymbol{r}_{k}}=\boldsymbol{r}_{k} / r_{k}$ with $r_{k}=\left|\boldsymbol{r}_{k}\right|$.

$T_{\alpha \beta}(\hat{\boldsymbol{r}})=\frac{3 r_{\alpha} r_{\beta}-r^{2} \delta_{\alpha \beta}}{r^{2}}$

is a traceless tensor expressed as

$T(\hat{\boldsymbol{r}})=\sqrt{\frac{4 \pi}{5}}\left(\begin{array}{ccc}-K_{2,4}(\hat{\boldsymbol{r}})+\sqrt{3} K_{2,5}(\hat{\boldsymbol{r}}) & \sqrt{3} K_{2,1}(\hat{\boldsymbol{r}}) & \sqrt{3} K_{2,3}(\hat{\boldsymbol{r}}) \\ \sqrt{3} K_{2,1}(\hat{\boldsymbol{r}}) & -K_{2,4}(\hat{\boldsymbol{r}})-\sqrt{3} K_{2,5}(\hat{\boldsymbol{r}}) & \sqrt{3} K_{2,2}(\hat{\boldsymbol{r}}) \\ \sqrt{3} K_{2,1}(\hat{\boldsymbol{r}}) & \sqrt{3} K_{2,2}(\hat{\boldsymbol{r}}) & 2 K_{2,4}(\hat{\boldsymbol{r}})\end{array}\right)$

with the cubic harmonics $K_{l, m}(\hat{\boldsymbol{r}})$; see Appendix A for the definition.

\section{B. Implementation}

We implement the calculation routine of HFI in the all-electron mixed basis code, TOMBO, in which both plane waves (PWs) and atomic orbitals (AOs) are used as basis functions. The AOs are constructed by a product of the numerical radial function in a logarithmic mesh and the analytic cubic harmonics. In particular for the valence AOs, the radial function is confined inside the nonoverlapping atomic sphere by subtracting a smooth quadratic function with the same amplitude and derivative at the atomic sphere ${ }^{25}$. This quadratic function inside the sphere smoothly connects to the tail of the true AO outside the sphere. Sum of this smoothly connected part and the truncated part is equal to the true AO. Then, the former can be well described by a linear combination of $\mathrm{PWs}$, and the latter is used as AO in the program.

The molecular orbitals are expanded as

$\phi_{\lambda}(\boldsymbol{r}, s)=\frac{1}{\sqrt{\Omega}} \sum_{\boldsymbol{G}} c_{\boldsymbol{G}}^{\lambda}(s) e^{i \boldsymbol{G} \cdot \boldsymbol{r}}+\sum_{k=1}^{N} \sum_{n l m} c_{k n l m}^{\lambda}(s) \varphi_{n l m}\left(\boldsymbol{r}-\boldsymbol{R}_{k}\right)$,

where $\Omega$ is the volume of the unit cell and AO is expressed as

$\varphi_{n l m}\left(\boldsymbol{r}_{k}\right)=R_{n l}\left(r_{k}\right) K_{l m}\left(\widehat{\boldsymbol{r}_{k}}\right)$ 
with $\boldsymbol{r}_{k}=\boldsymbol{r}-\boldsymbol{R}_{k}$. The electron density is decomposed into PW-PW, PW-AO, and AO-AO parts as $\rho(\boldsymbol{r}, s)=\sum_{\lambda}^{\mathrm{occ}}\left\{\left|\phi_{\lambda}(\boldsymbol{r}, s)\right|^{2}-\left|\phi_{\lambda}(\boldsymbol{r}, s)\right|^{2}\right\}=\rho^{\mathrm{PW}-\mathrm{PW}}(\boldsymbol{r}, s)+\rho^{\mathrm{PW}-\mathrm{AO}}(\boldsymbol{r}, s)+\rho^{\mathrm{AO}-\mathrm{AO}}(\boldsymbol{r}, s)$.

In the PW-AO part, PWs are first expanded in spherical waves around each $\boldsymbol{R}_{k}$ as

$e^{i \boldsymbol{G} \cdot \boldsymbol{r}}=4 \pi e^{i \boldsymbol{G} \cdot \boldsymbol{R}_{k}} \sum_{l=0}^{l_{\max }} i^{l} j_{l}\left(G r_{k}\right) \sum_{m} K_{l, m}(\widehat{\boldsymbol{G}}) K_{l, m}\left(\widehat{\boldsymbol{r}_{k}}\right)$,

where $l_{\max }$ is the maximum angular momentum to be taken into account. Then, both PW-AO and $\mathrm{AO}-\mathrm{AO}$ parts are represented by a linear combination of the biproducts of the cubic harmonics, which can be rewritten by a linear combination of the cubic harmonics using the coefficients given in Appendix A. The coefficients are similar to the Gaunt coefficients for the spherical harmonics:

$K_{l, m}\left(\widehat{\boldsymbol{r}_{k}}\right) K_{l^{\prime}, m^{\prime}}\left(\widehat{\boldsymbol{r}_{k}}\right)=\sum_{l^{\prime \prime}=0}^{l+l^{\prime}} \sum_{m^{\prime \prime}=1}^{2 l^{\prime \prime}+1} c_{l, m ; l^{\prime}, m^{\prime}}^{l^{\prime \prime}, m^{\prime \prime}} K_{l^{\prime \prime}, m^{\prime \prime}}\left(\widehat{\boldsymbol{r}_{k}}\right)$.

Then, using the orthonormality of the cubic harmonics

$\int K_{l, m}\left(\widehat{\boldsymbol{r}_{k}}\right) K_{l^{\prime}, m^{\prime}}\left(\widehat{\boldsymbol{r}_{k}}\right) d \widehat{\boldsymbol{r}_{k}}=\delta_{l l^{\prime}} \delta_{m m^{\prime}}$,

we can easily perform the integration appearing in Eq. (5), if we simply omit the AO contributions from other atoms ${ }^{22}$.

The AO contribution to the anisotropic, dipole interaction term Eq. (5), can be written as

$b_{k, \alpha \beta}^{\mathrm{AO}}=\frac{1}{4 \pi} \mu_{0} g_{\mathrm{e}} \mu_{\mathrm{e}} g_{k}\left\{\mu_{k} \int \frac{\rho_{s}^{\mathrm{AO}}\left(\boldsymbol{r}_{k}\right)}{r_{k}^{3}} T_{\alpha \beta}\left(\hat{\boldsymbol{r}}_{k}\right) d \boldsymbol{r}_{k}+\sum_{j \neq k)} \mu_{j} \int \frac{\rho_{s}^{\mathrm{AO}}\left(\boldsymbol{r}_{j}\right)}{\left|\boldsymbol{r}_{j}-\boldsymbol{R}_{j k}\right|^{3}} T_{\alpha \beta}\left(\widehat{\boldsymbol{r}_{j}-\boldsymbol{R}_{j k}}\right) d \boldsymbol{r}_{j}\right\}$,

where we wrote

$\rho_{s}^{\mathrm{AO}}\left(\boldsymbol{r}_{k}\right)= \begin{cases}\rho_{s}^{\mathrm{AO}-\mathrm{AO}}\left(\boldsymbol{r}_{k}+\boldsymbol{R}_{k}\right)+\rho_{s}^{\mathrm{PW}-\mathrm{AO}}\left(\boldsymbol{r}_{k}+\boldsymbol{R}_{k}\right), & \text { inside the } k \text {-th non-overlapping atomic sphere, } \\ 0 & \text { otherwise },\end{cases}$

and put $\boldsymbol{r}_{k}=\boldsymbol{r}-\boldsymbol{R}_{k}=\boldsymbol{r}_{j}-\boldsymbol{R}_{j k}, \boldsymbol{r}_{j}=\boldsymbol{r}-\boldsymbol{R}_{j}$, and $\boldsymbol{R}_{j k}=\boldsymbol{R}_{k}-\boldsymbol{R}_{j}$.

Let us consider to rewrite the cubic harmonics $T\left(\widehat{\boldsymbol{r}_{j}-\boldsymbol{R}_{j k}}\right)$ centered at $\boldsymbol{R}_{k}$ by the cubic harmonics $T\left(\widehat{\boldsymbol{r}_{j}}\right)$ centered at $\boldsymbol{R}_{j}$. As shown in Appendix B, $K_{L, M}\left(\widehat{\boldsymbol{r}_{j}-\boldsymbol{R}_{j k}}\right)$ can be rewritten in terms of $K_{L, M}\left(\widehat{\boldsymbol{r}_{j}}\right)$. Therefore, $T\left(\widehat{\boldsymbol{r}_{j}-\boldsymbol{R}_{j k}}\right)$ is rewritten in terms of $K_{L, M}\left(\widehat{\boldsymbol{r}_{j}}\right)$ as follows:

$T\left(\widehat{\boldsymbol{r}_{j}-\boldsymbol{R}_{j k}}\right)=\sqrt{\frac{4 \pi}{5}} \frac{1}{\left|\boldsymbol{r}_{j}-\boldsymbol{R}_{j k}\right|^{2}}\left\{r_{j}^{2}\left(\begin{array}{ccc}-K_{2,4}\left(\widehat{\boldsymbol{r}_{j}}\right)+\sqrt{3} K_{2,5}\left(\widehat{\boldsymbol{r}_{j}}\right) & \sqrt{3} K_{2,1}\left(\widehat{\boldsymbol{r}_{j}}\right) & \sqrt{3} K_{2,3}\left(\widehat{\boldsymbol{r}_{j}}\right) \\ \sqrt{3} K_{2,1}\left(\widehat{\boldsymbol{r}_{j}}\right) & -K_{2,4}-\sqrt{3} K_{2,5}\left(\widehat{\boldsymbol{r}_{j}}\right) & \sqrt{3} K_{2,2}\left(\widehat{\boldsymbol{r}_{j}}\right) \\ \sqrt{3} K_{2,3}\left(\widehat{\boldsymbol{r}_{j}}\right) & \sqrt{3} K_{2,2}\left(\widehat{\boldsymbol{r}_{j}}\right) & 2 K_{2,4}\left(\widehat{\boldsymbol{r}_{j}}\right)\end{array}\right)\right.$ 


$$
\begin{aligned}
& +r_{j} \sqrt{\frac{20}{3}}\left(\begin{array}{ccc}
2 Y K_{1,2}-X K_{1,1}-Z K_{1,3} & -\frac{3}{2}\left(Y K_{1,1}+X K_{1,2}\right) & -\frac{3}{2}\left(X K_{1,3}+Z K_{1,1}\right) \\
-\frac{3}{2}\left(Y K_{1,1}+X K_{1,2}\right) & 2 X K_{1,1}-Y K_{1,2}-Z K_{1,3} & -\frac{3}{2}\left(Z K_{1,2}+Y K_{1,3}\right) \\
-\frac{3}{2}\left(X K_{1,3}+Z K_{1,1}\right) & -\frac{3}{2}\left(Z K_{1,2}+Y K_{1,3}\right) & 2 Z K_{1,3}-X K_{1,1}-Y K_{1,2}
\end{array}\right)
\end{aligned}
$$

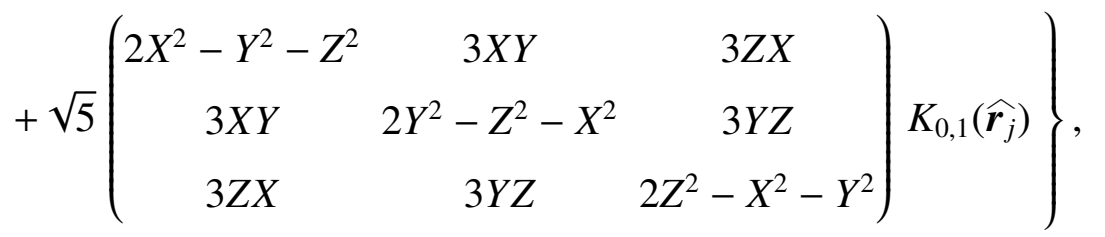

where we put $\boldsymbol{R}_{j k}=(X, Y, Z)$. Thus, the second term of Eq. (14) can be evaluated by the following three integrals:

$$
\int \frac{r_{j}^{2} \rho_{s}^{\mathrm{AO}}\left(\boldsymbol{r}_{j}\right) K_{2, M}\left(\widehat{\boldsymbol{r}_{j}}\right)}{\left|\boldsymbol{r}_{j}-\boldsymbol{R}_{j k}\right|^{5}} d \boldsymbol{r}_{j}, \quad R_{\alpha} \int \frac{r_{j} \rho_{s}^{\mathrm{AO}}\left(\boldsymbol{r}_{j}\right) K_{1, M}\left(\widehat{\boldsymbol{r}_{j}}\right)}{\left|\boldsymbol{r}_{j}-\boldsymbol{R}_{j k}\right|^{5}} d \boldsymbol{r}_{j}, \quad R_{\alpha} R_{\beta} \int \frac{\rho_{s}^{\mathrm{AO}}\left(\boldsymbol{r}_{j}\right) K_{0,1}\left(\widehat{\boldsymbol{r}_{j}}\right)}{\left|\boldsymbol{r}_{j}-\boldsymbol{R}_{j k}\right|^{5}} d \boldsymbol{r}_{j},
$$

where we put $R_{\alpha}, R_{\beta}=X, Y, Z(\alpha, \beta=1 \sim 3)$. Now we know that the contribution from surrounding atoms can be calculated by Eq. (17), the evaluation of Eq. (17) is not easy. Therefore, we introduce approximation. Since the interatomic distance $R_{j k}=\left|\boldsymbol{R}_{j k}\right|$ is larger than the root mean square radius of AOs, we use the approximation

$$
\frac{1}{\left|\boldsymbol{r}_{j}-\boldsymbol{R}_{j k}\right|^{5}} \approx \frac{1}{R_{j k}^{5}}
$$

Then, the denominator of Eq. (17) becomes constant and the calculation becomes easy. Thus the original (17) becomes

$$
\frac{1}{R_{j k}^{5}} \int r_{j}^{2} \rho_{s}^{\mathrm{AO}}\left(\boldsymbol{r}_{j}\right) K_{2, M}\left(\widehat{\boldsymbol{r}}_{j}\right) d \boldsymbol{r}_{j}, \quad \frac{R_{\alpha}}{R_{j k}^{5}} \int r_{j} \rho_{s}^{\mathrm{AO}}\left(\boldsymbol{r}_{j}\right) K_{1, M}\left(\widehat{\boldsymbol{r}}_{j}\right) d \boldsymbol{r}_{j}, \quad \frac{R_{\alpha} R_{\beta}}{R_{j k}^{5}} \int \rho_{s}^{\mathrm{AO}}\left(\boldsymbol{r}_{j}\right) K_{0,1}\left(\widehat{\boldsymbol{r}}_{j}\right) d \boldsymbol{r}_{j} .
$$

These terms can be calculated in a similar way as the on-site integral, i.e., the first term of Eq. (14). Only difference is that the power of $r$ is different. The power is -3 in the on-site integral, but it is now $0 \sim 2$. Because of $R^{5}$ in the denominator, the contribution from surrounding atoms is generally very small.

On the other hand, using the fast Fourier transformation (FFT), we can express the PW-PW part of the electron spin density in the following form:

$\rho_{s}^{\mathrm{PW}-\mathrm{PW}}(\boldsymbol{r})=\rho^{\mathrm{PW}-\mathrm{PW}}(\boldsymbol{r}, \uparrow)-\rho^{\mathrm{PW}-\mathrm{PW}}(\boldsymbol{r}, \downarrow)=\frac{1}{\Omega} \sum_{\boldsymbol{G}} f_{s}(\boldsymbol{G}) e^{i \boldsymbol{G} \cdot \boldsymbol{r}}$. 
Then, it is easy to perform the integration appearing in Eq. (5) as

$\int \frac{\rho_{s}^{\mathrm{PW}-\mathrm{PW}}\left(\boldsymbol{r}_{k}+\boldsymbol{R}_{k}\right)}{r_{k}^{3}} K_{l, m}\left(\widehat{\boldsymbol{r}_{k}}\right) d \boldsymbol{r}_{k}=\frac{4 \pi}{\Omega} \sum_{\boldsymbol{G}} f_{s}(\boldsymbol{G}) e^{i \boldsymbol{G} \cdot \boldsymbol{R}_{k}} K_{l, m}(\widehat{\boldsymbol{G}}) \int \frac{j_{l}\left(G r_{k}\right)}{r_{k}} d r_{k}: q:$

with $l=2$ and $m=1,2, \ldots, 5$.

\section{RESULTS AND DISCUSSION}

We have newly implemented the cubic harmonics HFI routines in the latest version of our all-electron mixed basis code, TOMBO, as described in the previous section, and applied it to the calculation of HFI for several spin-polarized systems using the local density approximation $(\mathrm{LDA})^{28}$ in density functional theory (DFT) to see the ability of the present method.

Figure 1 shows the dependence of the calculated isotropic, Fermi contact term $a_{k}$ on the (cubic) unit cell size for the fixed PW cutoff energy of $80 \mathrm{eV}$ for ${ }^{7} \mathrm{Li}$. The horizontal line is the experimental value ${ }^{29}$. From this figure, we see that the unit cell size of $12 \AA$ is enough to get an accurate result. We set the unit cell size at $12 \AA$ also for other systems. From similar convergence test, the PW cutoff energy of $80 \mathrm{eV}$ is used unless mentioned below.

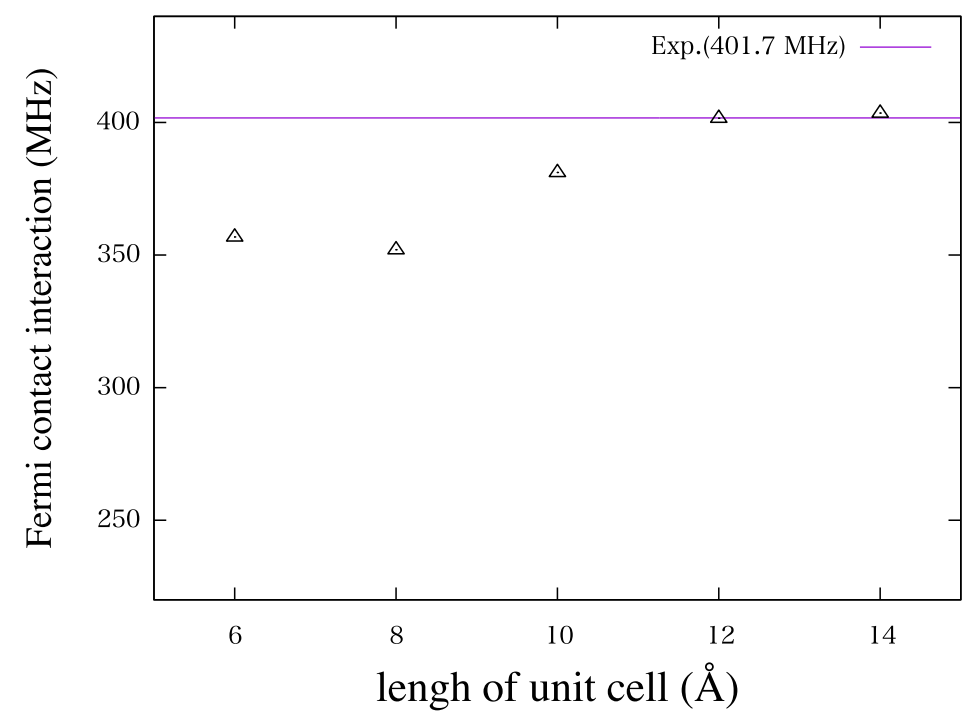

FIG. 1: Calculated isotropic, Fermi contact term of ${ }^{7} \mathrm{Li}$ (in units of $\mathrm{MHz}$ ) in different unit cell sizes (PW cutoff energy is $80 \mathrm{eV}$ )

Here, our method for the calculation of the isotropic, Fermi contact term of HFI is applied to 
group-I atoms $\left({ }^{7} \mathrm{Li},{ }^{23} \mathrm{Na},{ }^{39} \mathrm{~K}\right)$, group-II cations $\left({ }^{25} \mathrm{Mg}^{+},{ }^{43} \mathrm{Ca}^{+}\right)$and molecules $\left({ }^{67} \mathrm{Zn}^{1} \mathrm{H},{ }^{67} \mathrm{Zn}^{17} \mathrm{~F}\right)$. All these electronic states are ${ }^{2} \mathrm{~S}$ or ${ }^{2} \Sigma$.

The result of the isotropic, Fermi contact term for group-I atoms $\left({ }^{7} \mathrm{Li},{ }^{23} \mathrm{Na},{ }^{39} \mathrm{~K}\right)$ is shown in Table I. All these electronic states are ${ }^{2} \mathrm{~S}$. For comparison, the results of Bahramy et al. ${ }^{22}$, GAUSSIAN 03 (LSDA/DZVP) ${ }^{21}$, and the available experimental data are also listed in the same table. Our results agree well with the results of Bahramy et al. except for Na. Perhaps, the calculation of Na may need more careful check. The deviation from the experimental values is also small and is less than $2 \%$. The calculation error of estimate in our result is $1 \sim 2 \%$. The agreement with the experimental values seems slightly better than GAUSSIAN 03. Probably, using Gaussian orbitals is not easy to describe the cusp behavior at the nuclear position.

TABLE I: Comparison of the isotropic, Fermi contact term $a_{k}$ in units of MHz for group-I atoms

\begin{tabular}{|c|c|c|c|c|}
\hline \multirow[t]{2}{*}{ Atom } & \multirow[t]{2}{*}{ Present method } & \multirow{2}{*}{ Bahramy et al. ${ }^{\mathrm{a}}$} & GAUSSIAN $03^{b}$ & \multirow[t]{2}{*}{ Expt. } \\
\hline & & & LSDA/DZVP & \\
\hline${ }^{7} \mathrm{Li}$ & 401.6 & 401.6 & 382.7 & $401.752047(13)^{\mathrm{c}}$ \\
\hline${ }^{23} \mathrm{Na}$ & 902.2 & 891.2 & 905.7 & $885.8131(1)^{\mathrm{c}, \mathrm{d}}$ \\
\hline${ }^{39} \mathrm{~K}$ & 233.1 & 232.4 & 242.1 & $230.859845(15)^{\mathrm{c}}$ \\
\hline \multicolumn{5}{|c|}{${ }^{a}$ Ref. $^{22}$. } \\
\hline \multicolumn{5}{|c|}{${ }^{\mathrm{b}}$ Ref. $^{21}$. } \\
\hline \multicolumn{5}{|c|}{${ }^{\mathrm{c}}$ Refs. ${ }^{29,30}$. } \\
\hline${ }^{\mathrm{d}}$ Ref. $^{3}$ & & & & \\
\hline
\end{tabular}

Table II summarizes the results of the isotropic, Fermi contact term of the ${ }^{2} \mathrm{~S}$ state for group-II cations $\left({ }^{25} \mathrm{Mg}^{+},{ }^{43} \mathrm{Ca}^{+}\right)$of the present method, Bahramy et al. ${ }^{22}$, GAUSSIAN ${ }^{21,22}$, and the fourcomponent coupled-cluster (CC) method ${ }^{32}$ together with the experimental data. Results for ${ }^{25} \mathrm{Mg}^{+}$ and ${ }^{43} \mathrm{Ca}^{+}$are fairly in good agreement with experimental data. However, for ${ }^{87} \mathrm{Sr}^{+}$, the present method does not give a satisfactory result probably because we have completely ignored the relativistic effects and the change of the AOs due to the relativistic corrections.

Next, we calculated the anisotropic, dipole interaction term of HFI. First, we considered second row atoms, ${ }^{11} \mathrm{~B},{ }^{13} \mathrm{C}$ and ${ }^{17} \mathrm{O}$. Their electronic states are ${ }^{2} \mathrm{P},{ }^{3} \mathrm{P}$, and ${ }^{4} \mathrm{~S}$, respectively. We chose the PW cutoff energy of $200 \mathrm{eV}$ and the radius of the atomic sphere of $2.0 \AA$ in this calculation. The 
TABLE II: Comparison of the isotropic, Fermi contact term $a_{k}$ in units of $\mathrm{MHz}$ for group-II cations

\begin{tabular}{cccccc}
\hline \hline Cation & Present method & Bahramy et al. $^{\mathrm{a}}$ & GAUSSIAN & $\mathrm{CC}^{\mathrm{d}}$ & Expt. \\
\hline${ }^{25} \mathrm{Mg}^{+}$ & -601.7 & -600.2 & $-541.5^{\mathrm{b}}$ & -593.7 & $-596.254376(54)^{\mathrm{e}}$ \\
${ }^{43} \mathrm{Ca}^{+}$ & -825.0 & -812.8 & $-789.8^{\mathrm{c}}$ & -792.8 & $-806.40207160(8)^{\mathrm{f}}$ \\
${ }^{87} \mathrm{Sr}^{+}$ & -874.0 & -912.7 & $-829.0^{\mathrm{b}}$ & -1000 & $990-1000.5^{\mathrm{g}}$ \\
\hline \hline
\end{tabular}

${ }^{\mathrm{a}}$ Ref. $^{22}$.

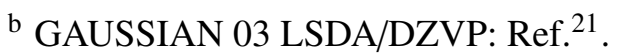

${ }^{c}$ GAUSSIAN 98 B3LYP/6-311+G(d): Ref. ${ }^{22}$.

${ }^{\mathrm{d}}$ Ref. $^{32}$.

${ }^{\mathrm{e}}$ Ref. $^{33}$.

${ }^{\mathrm{f}}$ Ref. $^{34}$.

${ }^{\mathrm{g}}$ Experimental data from Refs. ${ }^{21,35}$.

calculated results are summarized in Table III together with the previous results using B3LYP ${ }^{16}$ and coupled cluster model CCSD $(\mathrm{T})^{36}$, where the B3LYP calculation utilized special basis set which were optimized for the computation of hyperfine coupling constants. As seen in Eqs. (6) and (7), matrix $T$ is traceless $\left(T_{x x}+T_{y y}+T_{z z}=b_{k, x x}+b_{k, y y}+b_{k, z z}=0\right)$. Furthermore, since the systems have a spherical symmetry for atoms (or a cylindrical symmetry for linear molecules), the relation $T_{x x}=T_{y y}\left(b_{k, x x}=b_{k, y y}\right)$ holds. Therefore, we have $b_{k, \text { ani }} \equiv b_{k, \text { ani }, \|}-b_{k, \text { ani }, \perp}=b_{k, z z}-b_{k, x x}=3 b_{k, z z} / 2$ as the anisotropic term. According to Table III, we succeeded in obtaining the values similar to the other computational methods ${ }^{16,36}$.

TABLE III: Comparison of the anisotropic, dipole interaction term $b_{k \text {,ani }}=b_{k, \text { ani, } \|}-b_{k, \text { ani, } \perp}=3 b_{k, z z} / 2$ in units of $\mathrm{MHz}$ for second row atoms

\begin{tabular}{cccc}
\hline \hline Atom & Present method & B3LYP $^{\text {a }}$ & CCSD $(T) ~^{b}$ \\
\hline${ }^{11} \mathrm{~B}\left({ }^{2} \mathrm{P}\right)$ & 58.5 & 61.2 & 55.1 \\
${ }^{13} \mathrm{C}\left({ }^{3} \mathrm{P}\right)$ & 105.2 & 100.1 & 93.8 \\
${ }^{17} \mathrm{O}\left({ }^{4} \mathrm{~S}\right)$ & 152.9 & 152.2 & 150.4 \\
\hline \hline
\end{tabular}

${ }^{a}$ Ref. ${ }^{16}$

${ }^{b} \operatorname{Ref}^{36}$. 
Second, we applied our method to zinc compounds. The results for the ${ }^{2} \Sigma$ state are summarized in Table IV. In this calculation, the PW cutoff energy was set to $250 \mathrm{eV}$ for ${ }^{67} \mathrm{Zn}^{1} \mathrm{H}$ and $150 \mathrm{eV}$ for ${ }^{67} \mathrm{Zn}^{19} \mathrm{~F}$. Our results are comparable to the other methods ${ }^{21,22}$ and the available experimental data $^{38}$. We found that the experimental value for ${ }^{19} \mathrm{~F}$ of ${ }^{67} \mathrm{Zn}^{19} \mathrm{~F}$ was wrongly quoted in Ref. ${ }^{22}$, and the present method gives better agreement with this experiment, compared with the result of Bahramy et al. $^{22}$. For the anisotropic, dipole interaction term of $\mathrm{ZnF}$, for example, $b_{k \text {, ani }}$ for $k=\mathrm{Zn}$ has $3.03 \%$ contribution from the adjacent $\mathrm{F}$ atom and $b_{k \text {,ani }}$ for $k=\mathrm{F}$ has $8.08 \%$ contribution from the adjacent $\mathrm{Zn}$ atom. Therefore, the contributions from the second term of Eq. (14) is important and not negligible. The value of $b_{k \text {,ani }}$ for ${ }^{1} \mathrm{H}$ is remarkably small in comparison with other results in Table IV. From the view point of calculations, hydrogen atom generally has only small HFI contribution because it is basically not much spin polarized. Moreover, it could be either spherically symmetric or almost completely ionized. The error bars introduced by the approximation of Eq. (18) are less than the values given in Table IV.

TABLE IV: Comparison of the isotropic and anisotropic terms in units of $\mathrm{MHz}$ for $\mathrm{ZnH}$ and $\mathrm{ZnF}$ compounds

\begin{tabular}{|c|c|c|c|c|c|}
\hline \multirow[t]{2}{*}{ Molcule } & \multirow[t]{2}{*}{ Method } & \multicolumn{2}{|c|}{$\mathrm{Zn}$} & \multicolumn{2}{|c|}{$\mathrm{X}$} \\
\hline & & $a_{k}$ & $b_{k, \text { ani }}$ & $a_{k}$ & $b_{k, \text { ani }}$ \\
\hline \multirow[t]{4}{*}{${ }^{67} \mathrm{Zn}^{1} \mathrm{H}$} & Present method & 518.6 & 64.3 & 435.3 & -0.1 \\
\hline & Bahramy et al. a & 549.2 & 74.1 & 468.5 & -1.3 \\
\hline & GAUSSIAN 03 LSDA/DZVP b & 553.7 & 66.2 & 540.8 & -2.2 \\
\hline & Expt. ${ }^{c}$ & - & - & $478(3), 495(4)$ & $-1 \pm 2$ \\
\hline \multirow[t]{4}{*}{${ }^{67} \mathrm{Zn}^{19} \mathrm{~F}$} & Present method & 1146.1 & 25.1 & 358.1 & 820.7 \\
\hline & Bahramy et al. ${ }^{\text {a }}$ & 1151.6 & 60.5 & 196.2 & 822.6 \\
\hline & GAUSSIAN 03 LSDA/DZVP b & 1179.9 & 42.5 & 353.4 & 827.1 \\
\hline & Expt. ${ }^{\mathrm{d}}$ & - & - & $129(2), 320(2)$ & $816(4), 530(4)$ \\
\hline
\end{tabular}

\footnotetext{
${ }^{a}$ Ref. ${ }^{22}$.

b Ref. ${ }^{21}$.

c Ref. ${ }^{37}$.

${ }^{\mathrm{d}}$ Refs. ${ }^{38,39}$.
} 


\section{CONCLUSION}

In this work, we have implemented the cubic harmonics routines for calculating hyperfine interaction (HFI) in our all-electron mixed basis code, TOMBO. We presented the explicit coefficients (similar to the Gaunt coefficients for the spherical harmonics) when a biproduct of the cubic harmonics is expanded in a linear combination of the cubic harmonics in Appendix A. The results for the Fermi contact (isotropic) term of atoms and molecules are in good agreement with the available experimental data. The results for the dipole interaction term of atoms and $\mathrm{Zn}$ compounds are in fair agreement with the previous calculations of Perela et al. ${ }^{36}$ and available experimental data. In this calculation, we included the contributions from the surrounding atoms. All these results suggest the validity of the present method for calculating HFI. We are planning to apply the method to larger systems in the future.

\section{Appendix A}

The cubic harmonics are given by

$$
\begin{aligned}
& K_{0,1}=1, \quad K_{1,1}=\sqrt{3} x, \quad K_{1,1}=\sqrt{3} y, \quad K_{1,1}=\sqrt{3} z, \\
& K_{2,1}=\sqrt{15} x y, \quad K_{2,2}=\sqrt{15} y z, \quad K_{2,3}=\sqrt{15} z x, \quad K_{2,4}=\frac{\sqrt{5}}{2}\left(3 z^{2}-r^{2}\right), \quad K_{2,5}=\frac{\sqrt{15}}{2}\left(x^{2}-y^{2}\right),
\end{aligned}
$$

$$
\begin{aligned}
& K_{3,1}=\frac{\sqrt{7}}{2} x\left(5 x^{2}-3 r^{2}\right), \quad K_{3,2}=\frac{\sqrt{7}}{2} y\left(5 y^{2}-3 r^{2}\right), \quad K_{3,3}=\frac{\sqrt{7}}{2} z\left(5 z^{2}-3 r^{2}\right), \\
& K_{3,4}=\frac{\sqrt{105}}{2} x\left(y^{2}-z^{2}\right), \quad K_{3,5}=\frac{\sqrt{105}}{2} y\left(z^{2}-x^{2}\right), \quad K_{3,6}=\frac{\sqrt{105}}{2} z\left(x^{2}-y^{2}\right), \quad K_{3,7}=\sqrt{105} x y z
\end{aligned}
$$

$$
\begin{aligned}
& K_{4,1}=\frac{3 \sqrt{35}}{2} x y\left(x^{2}-y^{2}\right), \quad K_{4,2}=\frac{3 \sqrt{35}}{2} y z\left(y^{2}-z^{2}\right), \quad K_{4,3}=\frac{3 \sqrt{35}}{2} z x\left(z^{2}-x^{2}\right), \\
& K_{4,4}=\frac{3 \sqrt{5}}{2} x y\left(6 z^{2}-x^{2}-y^{2}\right), \quad K_{4,5}=\frac{3 \sqrt{5}}{2} y z\left(6 x^{2}-y^{2}-z^{2}\right), \quad K_{4,6}=\frac{3 \sqrt{5}}{2} z x\left(6 y^{2}-x^{2}-z^{2}\right), \\
& K_{4,7}=\frac{5 \sqrt{21}}{4}\left(x^{4}+y^{4}-z^{4}-\frac{3}{5} r^{4}\right), \quad K_{4,8}=\frac{7 \sqrt{15}}{4}\left[2 z^{4}-\left(x^{4}+y^{4}\right)-\frac{6}{7}\left(3 z^{2}-r^{2}\right) r^{2}\right] \\
& K_{4,9}=\frac{21 \sqrt{5}}{4}\left[x^{4}-y^{4}-\frac{6}{7}\left(x^{2}-y^{2}\right) r^{2}\right]
\end{aligned}
$$


except for a factor $1 /\left(\sqrt{4 \pi} r^{l}\right)$. They are related to the spherical harmonics by

$$
\begin{aligned}
& K_{0,1}=Y_{0,0}, \quad K_{11}=\frac{1}{\sqrt{2}}\left(Y_{1,1}-Y_{1,-1}\right), \quad K_{12}=\frac{i}{\sqrt{2}}\left(Y_{1,1}+Y_{1,-1}\right), \quad K_{13}=Y_{1,0}, \\
& K_{2,1}=\frac{1}{\sqrt{2} i}\left(Y_{2,2}-Y_{2,-2}\right), \quad K_{2,2}=\frac{1}{\sqrt{2} i}\left(Y_{2,1}-Y_{2,-1}\right), \quad K_{2,3}=\frac{1}{\sqrt{2}}\left(Y_{2,1}+Y_{2,-1}\right), \\
& K_{2,4}=Y_{2,0}, \quad K_{2,5}=\frac{1}{\sqrt{2}}\left(Y_{2,2}+Y_{2,-2}\right),
\end{aligned}
$$

and so forth.

It is well known that a biproduct of the spherical harmonics can be represented by a linear combination of the spherical harmonics. Its expansion coefficients are called the Gaunt coefficients. As well, a biproduct of the cubic harmonics can be represented by a linear combination of the cubic harmonics. The coeffiecents are calculated as

$$
\begin{aligned}
& K_{1,1} K_{1,2}=3 x y=\sqrt{\frac{3}{5}} K_{2,1}, \quad K_{1,2} K_{1,3}=3 y z=\sqrt{\frac{3}{5}} K_{2,2}, \quad K_{1,3} K_{1,1}=3 z x=\sqrt{\frac{3}{5}} K_{2,3}, \\
& K_{1,1} K_{1,1}=3 x^{2}=\sqrt{\frac{3}{5}} K_{2,5}-\frac{1}{\sqrt{5}} K_{2,4}+K_{0,1}, \\
& K_{1,2} K_{1,2}=3 y^{2}=-\sqrt{\frac{3}{5}} K_{2,5}-\frac{1}{\sqrt{5}} K_{2,4}+K_{0,1}, \quad K_{1,3} K_{1,3}=3 z^{2}=\frac{2}{\sqrt{5}} K_{2,4}+K_{0,1}
\end{aligned}
$$


for the product of $l=1$ and $l=1$,

$$
\begin{aligned}
& K_{1,1} K_{2,1}=3 \sqrt{5} x^{2} y=-\sqrt{\frac{3}{7}} K_{3,5}-\sqrt{\frac{3}{5}} K_{1,1}-\frac{3}{\sqrt{35}} K_{3,2} \\
& K_{1,1} K_{2,2}=3 \sqrt{5} x y z=\sqrt{\frac{3}{7}} K_{3,7}, \quad K_{1,1} K_{2,3}=3 \sqrt{5} x^{2} z=\sqrt{\frac{3}{7}} K_{3,6}+\sqrt{\frac{3}{5}} K_{1,3}-\frac{3}{\sqrt{35}} K_{3,3} \\
& K_{1,2} K_{2,2}=3 \sqrt{5} y^{2} z=-\sqrt{\frac{3}{7}} K_{3,6}+\sqrt{\frac{3}{5}} K_{1,3}-\frac{3}{\sqrt{35}} K_{3,3}, \quad K_{1,2} K_{2,3}=3 \sqrt{5} x y z=\sqrt{\frac{3}{7}} K_{3,7} \\
& K_{1,2} K_{2,1}=3 \sqrt{5} x y^{2}=\sqrt{\frac{3}{7}} K_{3,4}+\sqrt{\frac{3}{5}} K_{1,1}-\frac{3}{\sqrt{35}} K_{3,1}, \\
& K_{1,3} K_{2,3}=3 \sqrt{5} x z^{2}=-\sqrt{\frac{3}{7}} K_{3,4}+\sqrt{\frac{3}{5}} K_{1,1}-\frac{3}{\sqrt{35}} K_{3,1}, \\
& K_{1,3} K_{2,1}=3 \sqrt{5} x y z=\sqrt{\frac{3}{7}} K_{3,7}, \quad K_{1,3} K_{2,2}=3 \sqrt{5} y z^{2}=\sqrt{\frac{3}{7}} K_{3,5}+\sqrt{\frac{3}{5}} K_{1,2}-\frac{3}{\sqrt{35}} K_{3,2}, \\
& K_{1,3} K_{2,4}=\frac{\sqrt{15}}{2} z\left(3 z^{2}-r^{2}\right)=\frac{3 \sqrt{3}}{\sqrt{35}} K_{3,3}+\frac{2}{\sqrt{5}} K_{1,3}, \\
& K_{1,1} K_{2,4}=\frac{\sqrt{15}}{2} x\left(3 z^{2}-r^{2}\right)=-\frac{3}{2 \sqrt{7}} K_{3,4}+\frac{1}{\sqrt{5}} K_{1,1}-\frac{3 \sqrt{3}}{2 \sqrt{35}} K_{3,1}, \\
& K_{1,2} K_{2,4}=\frac{\sqrt{15}}{2} y\left(3 z^{2}-r^{2}\right)=\frac{3}{2 \sqrt{7}} K_{3,5}-\frac{1}{\sqrt{5}} K_{1,2}-\frac{3 \sqrt{3}}{2 \sqrt{35}} K_{3,2}, \\
& K_{1,1} K_{2,5}=\frac{3 \sqrt{5}}{2} x\left(x^{2}-y^{2}\right)=-\frac{\sqrt{3}}{2 \sqrt{7}} K_{3,4}-\sqrt{\frac{3}{5}} K_{1,1}-\frac{9}{2 \sqrt{35}} K_{3,1}, \\
& K_{1,5}=\frac{3 \sqrt{5}}{2} y\left(x^{2}-y^{2}\right)=-\frac{9}{2} K_{3,5}-\sqrt{\frac{3}{5}} K_{1,2}-\frac{9}{2} K_{3,2}, \\
& K_{3,6}
\end{aligned}
$$


for the product of $l=1$ and $l=2$, and

$$
\begin{aligned}
& K_{2,4} K_{2,4}=\frac{5}{4}\left(3 z^{2}-r^{2}\right)^{2}=\sqrt{\frac{3}{7}} K_{4,7}+\frac{\sqrt{15}}{7} K_{4,8}+K_{0,1}+\frac{2 \sqrt{5}}{7} K_{2,4}, \\
& K_{2,5} K_{2,5}=\frac{15}{4}\left(x^{2}-y^{2}\right)^{2}=\sqrt{\frac{3}{7}} K_{4,7}-\frac{\sqrt{15}}{7} K_{4,8}+K_{0,1}-\frac{2 \sqrt{5}}{7} K_{2,4}, \\
& K_{2,1} K_{2,1}=15 x^{2} y^{2}=-\frac{2}{\sqrt{21}} K_{4,7}-\frac{4 \sqrt{5}}{7 \sqrt{3}} K_{4,8}+K_{0,1}-\frac{2 \sqrt{5}}{7} K_{2,4}, \\
& K_{2,2} K_{2,2}=15 y^{2} z^{2}=\frac{2 \sqrt{5}}{7} K_{4,9}-\frac{2}{\sqrt{21}} K_{4,7}-\frac{2 \sqrt{5}}{7 \sqrt{3}} K_{4,8}+K_{0,1}+\frac{\sqrt{5}}{7} K_{2,4}-\frac{\sqrt{15}}{7} K_{2,5}, \\
& K_{2,3} K_{2,3}=15 z^{2} x^{2}=-\frac{2 \sqrt{5}}{7} K_{4,9}-\frac{2}{\sqrt{21}} K_{4,7}-\frac{2 \sqrt{5}}{7 \sqrt{3}} K_{4,8}+K_{0,1}+\frac{\sqrt{5}}{7} K_{2,4}+\frac{\sqrt{15}}{7} K_{2,5} \text {, } \\
& K_{2,1} K_{2,2}=15 z x y^{2}=\frac{\sqrt{15}}{7} K_{2,3}+\frac{2 \sqrt{5}}{7} K_{4,6}, \\
& K_{2,1} K_{2,3}=15 y z x^{2}=\frac{\sqrt{15}}{7} K_{2,2}+\frac{2 \sqrt{5}}{7} K_{4,5}, \\
& K_{2,2} K_{2,3}=15 x y z^{2}=\frac{\sqrt{15}}{7} K_{2,1}+\frac{2 \sqrt{5}}{7} K_{4,4}, \\
& K_{2,1} K_{2,5}=\frac{15}{2} x y\left(x^{2}-y^{2}\right)=\frac{\sqrt{15}}{7} K_{4,1}, \\
& K_{2,2} K_{2,5}=\frac{15}{2} y z\left(x^{2}-y^{2}\right)=-\frac{\sqrt{15}}{7} K_{2,2}+\frac{3 \sqrt{5}}{14} K_{4,5}-\frac{\sqrt{5}}{2 \sqrt{7}} K_{4,2} \text {, } \\
& K_{2,3} K_{2,5}=\frac{15}{2} z x\left(x^{2}-y^{2}\right)=\frac{\sqrt{15}}{7} K_{2,3}-\frac{3 \sqrt{5}}{14} K_{4,6}-\frac{\sqrt{5}}{2 \sqrt{7}} K_{4,3} \text {, } \\
& K_{2,1} K_{2,4}=\frac{5 \sqrt{3}}{2} x y\left(3 z^{2}-r^{2}\right)=-\frac{2 \sqrt{5}}{7} K_{2,1}+\frac{\sqrt{15}}{7} K_{4,5} \text {, } \\
& K_{2,2} K_{2,4}=\frac{5 \sqrt{3}}{2} y z\left(3 z^{2}-r^{2}\right)=\frac{\sqrt{5}}{7} K_{2,2}-\frac{\sqrt{15}}{14} K_{4,5}-\frac{\sqrt{15}}{2 \sqrt{7}} K_{4,2}, \\
& K_{2,3} K_{2,4}=\frac{5 \sqrt{3}}{2} z x\left(3 z^{2}-r^{2}\right)=\frac{\sqrt{5}}{7} K_{2,3}-\frac{\sqrt{15}}{14} K_{4,6}+\frac{\sqrt{15}}{2 \sqrt{7}} K_{4,3}
\end{aligned}
$$

for the product of $l=2$ and $l=2$.

\section{Appendix B}

Here we consider to rewrite the cubic harmonics $K_{L M}(\widehat{\boldsymbol{r}-\boldsymbol{R}})$ centered at $(\boldsymbol{r}-\boldsymbol{R})$ in terms of the cubic harmonics $K_{L M}(\hat{r})$ centered at $\boldsymbol{r}$. First of all, we define the relative coordinate from the basis atom as

$\boldsymbol{R}=(X, Y, Z)$ 
Using this and through elementary algebra

$$
\begin{aligned}
K_{2,1}(\widehat{\boldsymbol{r}-\boldsymbol{R}}) & =\sqrt{\frac{15}{4 \pi}} \frac{(x-X)(y-Y)}{|\boldsymbol{r}-\boldsymbol{R}|^{2}} \\
& =\sqrt{\frac{15}{4 \pi}} \frac{x y-Y x-X y+X Y}{|\boldsymbol{r}-\boldsymbol{R}|^{2}} \\
& =\frac{1}{|\boldsymbol{r}-\boldsymbol{R}|^{2}}\left\{r^{2} \sqrt{\frac{15}{4 \pi}} \frac{x y}{r^{2}}-r \sqrt{5} Y \sqrt{\frac{3}{4 \pi}} \frac{x}{r}-r \sqrt{5} X \sqrt{\frac{3}{4 \pi}} \frac{y}{r}+\sqrt{15} X Y \frac{1}{\sqrt{4 \pi}}\right\} \\
& =\frac{r^{2} K_{2,1}(\hat{\boldsymbol{r}})-r \sqrt{5} Y K_{1,1}(\hat{\boldsymbol{r}})-r \sqrt{5} X K_{1,2}(\hat{\boldsymbol{r}})+\sqrt{15} X Y K_{0,1}(\hat{\boldsymbol{r}})}{|\boldsymbol{r}-\boldsymbol{R}|^{2}}
\end{aligned}
$$

$$
\begin{aligned}
K_{2,2}(\widehat{\boldsymbol{r}-\boldsymbol{R}}) & =\sqrt{\frac{15}{4 \pi}} \frac{(y-Y)(z-Z)}{|\boldsymbol{r}-\boldsymbol{R}|^{2}} \\
& =\sqrt{\frac{15}{4 \pi}} \frac{y z-Z y-Y z+Y Z}{|\boldsymbol{r}-\boldsymbol{R}|^{2}} \\
& =\frac{1}{|\boldsymbol{r}-\boldsymbol{R}|^{2}}\left\{r^{2} \sqrt{\frac{15}{4 \pi}} \frac{y z}{r^{2}}-r \sqrt{5} Z \sqrt{\frac{3}{4 \pi}} \frac{y}{r}-r \sqrt{5} Y \sqrt{\frac{3}{4 \pi}} \frac{z}{r}+\sqrt{15} Y Z \frac{1}{\sqrt{4 \pi}}\right\} \\
& =\frac{r^{2} K_{2,2}(\hat{\boldsymbol{r}})-r \sqrt{5} Z K_{1,2}(\hat{\boldsymbol{r}})-r \sqrt{5} Y K_{1,3}(\hat{\boldsymbol{r}})+\sqrt{15} Y Z K_{0,1}(\hat{\boldsymbol{r}})}{|\boldsymbol{r}-\boldsymbol{R}|^{2}}
\end{aligned}
$$

$$
\begin{aligned}
K_{2,3}(\widehat{\boldsymbol{r}-\boldsymbol{R}}) & =\sqrt{\frac{15}{4 \pi}} \frac{(z-Z)(x-X)}{|\boldsymbol{r}-\boldsymbol{R}|^{2}} \\
& =\sqrt{\frac{15}{4 \pi}} \frac{z x-X z-Z x+Z X}{|\boldsymbol{r}-\boldsymbol{R}|^{2}} \\
& =\frac{1}{|\boldsymbol{r}-\boldsymbol{R}|^{2}}\left\{r^{2} \sqrt{\frac{15}{4 \pi}} \frac{z x}{r^{2}}-r \sqrt{5} X \sqrt{\frac{3}{4 \pi}} \frac{z}{r}-r \sqrt{5} Z \sqrt{\frac{3}{4 \pi}} \frac{x}{r}+\sqrt{15} Z X \frac{1}{\sqrt{4 \pi}}\right\} \\
& =\frac{r^{2} K_{2,3}(\hat{\boldsymbol{r}})-r \sqrt{5} X K_{1,3}(\hat{\boldsymbol{r}})-r \sqrt{5} Z K_{1,1}(\hat{\boldsymbol{r}})+\sqrt{15} Z X K_{0,1}(\hat{\boldsymbol{r}})}{|\boldsymbol{r}-\boldsymbol{R}|^{2}}
\end{aligned}
$$

$$
\begin{aligned}
K_{2,4}(\widehat{\boldsymbol{r}-\boldsymbol{R}}) & =\sqrt{\frac{5}{16 \pi}} \frac{2(z-Z)^{2}-(x-X)^{2}-(y-Y)^{2}}{|\boldsymbol{r}-\boldsymbol{R}|^{2}} \\
& =\sqrt{\frac{5}{16 \pi}} \frac{2 z^{2}-x^{2}-y^{2}+4 Z z-2 X x-2 Y y+2 Z^{2}-X^{2}-Y^{2}}{|\boldsymbol{r}-\boldsymbol{R}|^{2}} \\
& =\frac{1}{|\boldsymbol{r}-\boldsymbol{R}|^{2}}\left\{r^{2} \sqrt{\frac{5}{16 \pi}} \frac{\left(2 z^{2}-x^{2}-y^{2}\right)}{r^{2}}+r \sqrt{\frac{20}{3}} Z \sqrt{\frac{3}{4 \pi}} \frac{z}{r}-r \sqrt{\frac{5}{3}} X \sqrt{\frac{3}{4 \pi}} \frac{x}{r}-r \sqrt{\frac{5}{3}} Y \sqrt{\frac{3}{4 \pi}} \frac{y}{r}\right\}
\end{aligned}
$$




$$
\begin{aligned}
+ & \frac{1}{|\boldsymbol{r}-\boldsymbol{R}|^{2}}\left\{\frac{\sqrt{5}}{2}\left(2 Z^{2}-X^{2}-Y^{2}\right) \frac{1}{\sqrt{4 \pi}}\right\} \\
& =\frac{r^{2} K_{2,4}(\hat{\boldsymbol{r}})+r \sqrt{\frac{20}{3}} Z K_{1,3}(\hat{\boldsymbol{r}})-r \sqrt{\frac{5}{3}} X K_{1,1}(\hat{\boldsymbol{r}})-r \sqrt{\frac{5}{3}} Y K_{1,2}(\hat{\boldsymbol{r}})}{|\boldsymbol{r}-\boldsymbol{R}|^{2}} \\
& +\frac{\sqrt{5}}{2|\boldsymbol{r}-\boldsymbol{R}|^{2}}\left(2 Z^{2}-X^{2}-Y^{2}\right) K_{0,1}(\hat{\boldsymbol{r}}) \\
K_{2,5}(\widehat{\boldsymbol{r}-\boldsymbol{R}}) & =\sqrt{\frac{15}{16 \pi}} \frac{(x-X)^{2}-(y-Y)^{2}}{|\boldsymbol{r}-\boldsymbol{R}|^{2}} \\
& =\sqrt{\frac{15}{16 \pi}} \frac{x^{2}-y^{2}-2 X x+2 Y y+X^{2}-Y^{2}}{|\boldsymbol{r}-\boldsymbol{R}|^{2}} \\
& =\frac{1}{|\boldsymbol{r}-\boldsymbol{R}|^{2}}\left\{r^{2} \sqrt{\frac{15}{16 \pi}} \frac{x^{2}-y^{2}}{r^{2}}-r \sqrt{5} X \sqrt{\frac{3}{4 \pi}} \frac{x}{r}+r \sqrt{5} Y \sqrt{\frac{3}{4 \pi}} \frac{y}{r}+\frac{\sqrt{15}}{2}\left(X^{2}-Y^{2}\right) \frac{1}{\sqrt{4 \pi}}\right\} \\
& =\frac{1}{|\boldsymbol{r}-\boldsymbol{R}|^{2}}\left\{r^{2} K_{2,5}(\hat{\boldsymbol{r}})-r \sqrt{5} X K_{1,1}(\hat{\boldsymbol{r}})+r \sqrt{5} Y K_{1,2}(\hat{\boldsymbol{r}})+\frac{\sqrt{15}}{2}\left(X^{2}-Y^{2}\right) K_{0,1}(\hat{\boldsymbol{r}})\right\}
\end{aligned}
$$

\section{Acknowledgments}

This work was supported by the Grant-in-Aid Scientific Research B (Grant No. 18H01939) form JSPS. We have been also indebted to the HPCI social and scientific priority issue "Creation of new functional devices and high-performance materials to support next-generation industries" to be tackled by using post-K computer promoted by MEXT for the use of the supercomputer facilities at the Institute for Solid State Physics, the University of Tokyo, at Hokkaido University, and at the Institute for Materials Research, Tohoku University (Project IDs. hp170268, hp170190, hp180125, and hp180220).

* ohno@ynu.ac.jp

1 E. Widmann, J. Eades, T. Ishikawa, J. Sakaguchi, T. Tasaki, H. Yamaguchi, R. S. Hayano, M. Hori, H.

A. Torii, B. Juhász, D. Horváth, and T. Yamazaki, Phys. Rev. Lett. 89, 243402 (2002).

2 S. Friedreich, D. Barna, F. Caspers, A. Dax, R. Hayano, M. Hori, D. Horváth D, B. Juház, T. Kobayashi, O. Massiczek, A. Sótér, K. Todoroki, E. Widmann, and J. Zmeskal, Hyperfine Interactions 212, 167 (2012). 
3 T. Shida, Y. Egawa, H. Kubodera, and T. Kato, J. Chem. Phys. 73, 5963 (1980).

${ }^{4}$ L. A. Eriksson, V. G. Malkin, and D. R. Salahub, J. Chem. Phys. 99, 9756 (1993).

5 M. Kaupp, M. Bühl, and V. G. Malkin, Calculation of NMR and EPR Parameters, Wiley-VCH, Weinheim, 2004.

6 P.J. Schurer, Z. Celinski, B. Heinrich, Phys. Rev. B 51, 2506 (1995).

7 U. Runge, M. Dippel, G. Fillebock, K. Jacobs, U. Kohl, and G. Schatz, Phys. Rev. Lett. 79, 3054 (1997).

8 C. O. Rodriguez, M. V. Ganduglia-Pirovano, E. L. P. y Blancá, and M. Petersen, Phys. Rev. B 64, 144419 (2001).

9 J. N. Gonçalves, and V. S. Amaral, Phys. Rev. B 83, 104421 (2011).

10 M. Schaffry, V. Filidou, S.D. Karlen, E. M. Gauger, S. C. Benjamin, H.L. Anderson, A. Ardavan, G.A.D. Briggs, K. Maeda, K.B. Henbest, F. Giustino, J.J.L. Morton, and B.W. Lovett, Phys. Rev. Lett. 104, 200501 (2010).

11 V. Filidou, D. Ceresoli, J. J. L. Morton, and F. Giustino, Phys. Rev. B 85, 115430 (2012).

12 W. Pfaff, B. J. Hensen, H. Bernien, S. B. van Dam, M. S. Blok, T. H. Taminiau, M.J. Tiggelman, R.N. Schouten, M. Markham, D.J. Twitchen, and R. Hanson, Science 345, 532 (2014).

13 A. Gali, M. Fyta, and E. Kaxiras, Phys. Rev. B 77, 155206 (2008).

14 C. G. Van de Walle, and P. E. Blöchl, Phys. Rev. B 47, 4244 (1993).

15 C. G. Van de Walle, and L. Pavesi, Phys. Rev. B 47, 4256 (1993).

16 V. Barone, J. Chem. Phys. 101, 6834 (1994).

17 R. H. Luchsinger, Y. Zhou, and P. F. Meier, Phys. Rev. B 55, 6927 (1997).

18 Z. Zeng, Y. Duan, and D. Guenzburger, Phys. Rev. B 55, 12522 (1997).

19 J. Lægsgaard, and K. Stokbro, Phys. Rev. B 65, 075208 (2001).

20 P. E. Blöchl, Phys. Rev. B 62, 6158 (2000).

21 R. Declerck, E. Pauwels, V. Van Speybroeck, and M. Waroquier, Phys. Rev. B 74, 245103 (2006).

22 M. S. Bahramy, M. H. F. Sluiter, and Y. Kawazoe, Phys. Rev. B 73, 045111 (2006).

23 C. T. White, F.W. Kutzler, and M. Cook, Phys. Rev. Lett. 56, 252 (1986).

24 K. Ohno, F. Mauri, and S. G. Louie, Phys. Rev. B 56, 1009 (1997)

25 S. Ono, Y. Noguchi, R. Sahara, Y. Kawazoe, and K. Ohno, Comp. Phys. Comm. 189, 20 (2015).

26 R. Kuwahara, Y. Noguchi, and K. Ohno, Phys. Rev. B 94, 121116(R) (2016).

27 T. Isobe, R. Kuwahara, and K. Ohno, Phys. Rev. A 97, 060502(R) (2018).

28 J. P. Perdew and A. Zunger, Phys. Rev. B 23, 5048 (1981). 
29 G.H. Fuller and V.W. Cohen, Nucl. Data Tables A 5, 433 (1969).

30 G. H. Fuller, J. Phys. Chem. Ref. Data 5, 835 (1969); The values listed in Table H in pp.952-953 of this paper must be divided by 2 .

31 M. Arditi and T. R. Carver, Phys. Rev. 109, 1012 (1958).

32 I. Malkin, O. L. Malkin, V. G. Malkin and M. Kaupp, Chem. Phys. Lett. 396, 268 (2004).

33 W. M. Itano and D. J. Wineland, Phys. Rev. A 24, 1364 (1981).

34 F. Arbes, M. Benzing, Th. Gudjons, F. Kurth, and G. Werth, Z. Phys. D 31, 27 (1994).

35 R. Beigang, W. Makat, A. Timmermann, and P. J. West, Phys. Rev, Lett. 51, 771 (1983).

36 S. A. Perera, J. D. Watts, and R. J. Bartlett, J. Chem. Phys. 100, 1425 (1994).

37 L. B. Knight Jr. and W. Weltner Jr., J. Chem. Phys. 55, 2061 (1971).

38 L. B. Knight Jr, A. Mouchet, W. T. Beaudry, and M. Duncan, J. Mag. Res. 32, 383 (1978).

39 W. Weltner Jr., Magnetic Atoms and Molecules, Van Nostrand Reinhold, New York, 1983. 